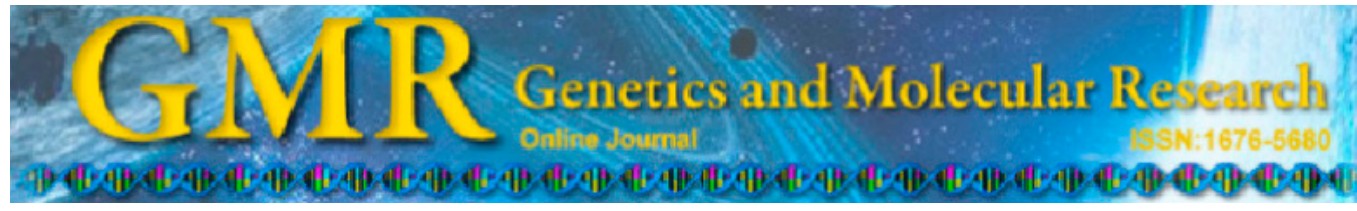

Short Communication

\title{
Huwe1 as a therapeutic target for neural injury
}

\author{
J. Zhou ${ }^{4}$, Q. Liu ${ }^{1,2}$, M. Mao ${ }^{1,2,3}$ and Y. Tong ${ }^{1,2}$ \\ ${ }^{1}$ Laboratory of Early Developmental and Injuries, \\ West China Institute of Woman and Children's Health, \\ West China Second University Hospital, Sichuan University, Chengdu, China \\ ${ }^{2}$ Key Laboratory of Obstetric \& Gynecologic and Pediatric Diseases and Birth \\ Defects of Ministry of Education, Chengdu, China \\ ${ }^{3}$ Department of Pediatrics, \\ West China Second University Hospital, Sichuan University, Chengdu, China \\ ${ }^{4}$ Department of Laboratory Medicine, West China Hospital, \\ Sichuan University, Chengdu, China \\ Corresponding author: Y. Tong \\ E-mail: zisu yu@163.com
}

Genet. Mol. Res. 13 (2): 4320-4325 (2014)

Received October 18, 2013

Accepted December 18, 2013

Published June 9, 2014

DOI http://dx.doi.org/10.4238/2014.June.9.18

ABSTRACT. The ubiquitin-proteasome system (UPS) regulates many
cellular processes, including protein stability, cell cycle control, DNA
repair, transcription, signal transduction, and protein trafficking. In fact,
UPS plays a key role in various stress conditions such as ischemia,
glutamate toxicity, Alzheimer's disease, and Parkinson's disease.
Huwe1, a homologous to E6-AP carboxy terminus (HECT) domain
ubiquitin ligase, is now being regarded as a vital protein involved
in neural stem cell differentiation, adult neurogenesis, and the DNA
damage response pathway. In response to DNA damage, Huwe1
may have a dual function in arresting DNA replication and in ending
checkpoint signaling. The proliferation and differentiation of neural
stem cells regulated by Huwe1-mediated Notch signaling could also
play an important role in neural protection following neural injury.
Considering Huwe1 is required for neural precursor survival and the
regulation of the DNA damage response pathway, there is growing 
evidence and considerable hope that Huwe1 might be a therapeutic target for neural injury.

Key words: Huwe1; DNA damage; Notch signaling; Neural stem cells

\section{INTRODUCTION}

Neural cells suffer functional or sensory loss in both neurodegenerative diseases (Wang et al., 2009; Palop and Mucke, 2010), such as Alzheimer's disease, Parkinson's disease, and multiple sclerosis, and acquired brain injuries, such as hypoxia-ischemia, stoke, and traumatic brain injury (Giza et al., 2009). Apart from several other environmental or genetic factors, impairment of the ubiquitin-proteasome system (UPS) is reported to have a calamitous role in neural injury, leading to a range of disorders such as Alzheimer's disease, Parkinson's disease, aging, and other neural disorders. Huwe1, a homologous to E6-AP carboxy terminus (HECT) domain ubiquitin ligase, is now being regarded as a vital protein involved in neural stem cell (NSC) differentiation, adult neurogenesis, and the DNA damage response pathway. Thus, a detailed understanding of the function of Huwe1 at the molecular, cellular, and systematic levels may provide insight into the neural self-protection mechanism during neural injury or disease. Here, we highlight the significance of recent Huwel findings in this field and propose that Huwe1 may play critical roles in nervous system plasticity, regeneration, and disease.

\section{UPS AND HUWE1}

Many cellular processes are regulated by UPS, including protein stability, cell cycle control, DNA repair, transcription, signal transduction, and protein trafficking (Kinyamu et al., 2005; Fasanaro et al., 2010; Willis et al., 2010). In fact, UPS plays a key role in various stress conditions such as ischemia, glutamate toxicity, Alzheimer's disease and Parkinson's disease (Valera et al., 2005; Meller, 2009; Jiang et al., 2010). The accumulation of unfolded and/or non-functional proteins in neurons is considered the hallmark of proteasomal dysfunction in neurodegenerative disorders (Dantuma and Lindsten, 2010). However, recent studies have also indicated the involvement of UPS in these diseases. In addition to neurodegenerative disorders, UPS dysfunction has also been described in the ischemic brain (Meller et al., 2008; Meller, 2009). For example, Yamauchi et al. (2008) reported the accumulation of mutant ubiquitin protein in dying CA1 neurons after ischemia, suggesting that UPS may be required for regulating neuronal survival during neuronal injury.

The UPS is an enzymatic cascade consisting of ubiquitin activating enzyme (E1), ubiquitin conjugating enzyme (E2), and ubiquitin ligase (E3), which is responsible for catalyzing ubiquitin conjugation to target proteins. The relevance of E3 in several biological processes has been emphasized in vivo by the observation that their genetic alteration, abnormal expression, or dysfunction is often accompanied by the occurrence of pathological disorders. There are two major classes of E3. One E3 class ( $~ 800$ human genes), contains a conserved RING finger motif and functions by binding both the substrate and E2, and activating E2's conjugating activity. Another class ( $\sim 90$ human genes), contains a conserved C-terminal HECT domain (named after E6AP, the first E3 described in this class) and functions by first accepting ubiq- 
uitin from E2 onto a cysteine residue and then conjugating the ubiquitin to the substrate (Maspero et al., 2013). The key signature of this E3 subfamily is the HECT domain (HECT E3s), which associates with E2 and provides the catalytic E3 activity (Kim and Huibregtse, 2009).

In a recent study, a major protein band of $\sim 500 \mathrm{kDa}$, named Huwe1 (also called ARF-BP1, Mule, Lasu1, Ureb1, E3 histone, and HectH9) was purified from HA-ARF-Flagexpressing H1299 cells by mass spectrometric analysis. Huwel encodes a HECT domain ubiquitin ligase, which has attracted considerable interest because several disparate substrates, such as p53, Mcl-1, Cdc6, and N-myc have been assigned to this E3. Although both in vitro and in vivo data indicated that Huwe1 is important for neurogenesis in cerebral cortex and possibly brain cancer, the exact biological function and underlying mechanism of Huwe1 remains controversial.

\section{DNA DAMAGE AND HUWE1}

During normal cell growth, Huwe1 ubiquitinates p53 to induce p53 degradation (Chen et al., 2005). In response to DNA damage, Huwe1 ubiquitinates Mcl-1 to induce its degradation, but no longer ubiquitinates p53, resulting in low Mcl-1 with stabilized p53, thus promoting cell cycle arrest and apoptosis. Among the anti-apoptotic members of the Bcl-2 protein family, Mcl-1 is unique because it is an early-response gene that is rapidly induced and turned over. This property enables Mcl-1 to function at an early step in the signaling cascade consisting of Bcl-2 family proteins, and provides an acute protective function against apoptosis induced by a variety of stimuli including DNA damage, adenoviral infection, growth factor withdrawal, and treatment of cytotoxic agents. Interestingly, Huwe1 also contains a well-conserved $\mathrm{BH} 3$ domain, which allows the Bcl-2 family members to interact with each other. Among the Bcl-2 family members, the BH3 domain of Huwe1 mostly resembles the $\mathrm{BH} 3$ domain of Bak, which is associated with Mcl-1 (Cuconati et al., 2003). It is conceivable that Huwe1 not only binds to free Mcl-1 through its BH3 domain, but also competes with Bak to break up the Mcl-1/Bak complex and promote Mcl-1 degradation (Zhong et al., 2005). In addition, Huwe1 binds to Cdc6 and topoisomerase II binding protein 1 (TopBP1) and degrades them in response to DNA damage (Hall et al., 2007). Cdc6 is not only an essential factor for prereplication complex (preRC, a multiprotein complex, an origin needed for DNA replication), but it is also implicated in the activation of the cell cycle checkpoint that prevents entry into mitosis when DNA replication is incomplete (Borlado and Mendez, 2008). TopBP1 is required for Cdc45 to the replication origins during DNA replication (Makiniemi et al., 2001). However, the time points at which Cdc6 and TopBP1 are degraded appear to be different, as Cdc6 degrades rapidly, but TopBP1 degradation is delayed after UV irradiation, suggesting that Huwe1 may have dual functions in arresting DNA replication and in ending checkpoint signaling after DNA damage (Herold et al., 2008).

Recently, Parsons et al. (2009) provided a new mechanism underlying the cellular response to DNA damage. It is known that base excision repair (BER) is the major cellular system involved in the removal of DNA lesions induced by endogenous and exogenous DNA damaging agents. Among the BER proteins, DNA polymerase $\beta$ (Pol $\beta$ ) has a central role in filling the nucleotide gap that arises during the repair process. The regulation of cellular Pol $\beta$ levels is vital, as under or overproduction of Pol $\beta$ leads to deficient repair or an increased 
rate of mutagenesis, respectively, and both have been linked to increased cancer susceptibility (Matsuda et al., 2003). Parsons et al. (2009) conceived that the dynamics of cellular Pol $\beta$ are controlled by Huwe1 and ARF, which determine the fate of the newly synthesized cytoplasmic Pol $\beta$. Huwe1 is predominantly a cytoplasmic protein, whereas ARF is located in the nucleoli. In response to DNA damage, ARF is released from the nucleoli, allowing it to interact with Huwe 1 and inhibiting its ubiquitin ligase activity, which restrains the degradation of Pol $\beta$ by Huwe1 (Parsons et al., 2009). These results provide a molecular link between DNA damage and Huwel activity.

\section{NSCs AND HUWE1}

There is growing evidence that neuronal injury and death act as triggers for NSC proliferation (Schwarting et al., 2008; Christie et al., 2013) and adult neurogenesis (Blaiss et al., 2011; Omar Chohan et al., 2013). Notch signaling is a key pathway for regulating the proliferation and differentiation of NSCs and progenitors. In the developing brain, activated Notch signaling maintains NSCs, promotes proliferation of neural progenitors, and inhibits progenitor differentiation into neurons (Mizutani et al., 2007). Recent data have demonstrated that Notch is also expressed in the adult mammalian hippocampus and anterior subventricular zone (aSVZ) (Givogri et al., 2006). Based on these previous studies, Oya et al. (2009) investigated the role of Notch signaling in the hippocampal CA1 region after transient global ischemia in rats, and found that the attenuation of Notch signaling in the subacute phase enhanced neuronal regeneration. It remains unclear whether Notch has any further effects during the later stages of differentiation and maturation. However, it was shown that activated Notch signaling inhibited neurite outgrowth in vitro, and that the signaling blockade promoted neurite development (Redmond et al., 2000). This suggests that modulation of Notch signaling in the later stages might be a therapeutic target for facilitating neuronal regeneration after brain injury in adults. In fact, Huwe1 is involved in the Notch signaling pathway. Zhao et al. (2009) conditionally deleted the Huwel gene in the mouse brain and demonstrated that this enzyme is essential for the transition from self-renewing and proliferating neural stem/ progenitor cells to differentiated neurons. Consequently, loss of Huwe 1 severely perturbs neurogenesis causing a disorganized laminar structure in the cortex. They also identified the "N-Myc-DLL3" pathway as a mediator of the abnormalities detected in the Huwe1null brain (Zhao et al., 2009). Recently, D'Arca et al. (2010) generated a knockout mouse strain in which the gene coding for the ubiquitin ligase Huwe1 is inactivated in cerebellar granule neuron precursors (CGNPs) and radial glia. Using this mouse strain, they demonstrated that Huwel plays a key role in the differentiation of CGNPs and maturation of Bergmann glia during the postnatal development of the cerebellum. Detailed analysis showed that the proliferative window of Huwe1-null granule neurons was prolonged, associated with elevated N-Myc and cyclin D2, and reduced p18Ink4C and p27Kip1 (D'Arca et al., 2010). This study established the essential role Huwe1 ubiquitin ligase plays in coordinating the maturation of neurons and glia in the nervous system. These previous studies suggest that the proliferation and differentiation of NSCs regulated by Huwe 1-mediated Notch signaling could also play an important role in neural protection following neural injury. 


\section{CONCLUSIONS}

DNA damage is implicated in the pathophysiology of a wide range of central nervous system diseases, including both acute injury and chronic neurodegenerative disorders. Because of their ability to integrate into the mature central nervous system, NSCs are thought to have potential for structural brain repair. Considering Huwe1 is required for neural precursor survival and the regulation of the DNA damage response pathway, there is now growing evidence and hope that Huwe1 might be a therapeutic target for neural injury.

\section{ACKNOWLEDGMENTS}

Research supported by the National Natural Science Foundation of China (\#0973215), Sichuan Province Science and Technology Foundation (\#2012SZ0010), and the Program of Changjiang Scholars and Innovative Research Team in University (IRT0935).

\section{REFERENCES}

Blaiss CA, Yu TS, Zhang G, Chen J, et al. (2011). Temporally specified genetic ablation of neurogenesis impairs cognitive recovery after traumatic brain injury. J. Neurosci. 31: 4906-4916.

Borlado LR and Mendez J (2008). CDC6: from DNA replication to cell cycle checkpoints and oncogenesis. Carcinogenesis 29: 237-243.

Chen D, Kon N, Li M, Zhang W, et al. (2005). ARF-BP1/Mule is a critical mediator of the ARF tumor suppressor. Cell 121: 1071-1083.

Christie KJ, Emery B, Denham M, Bujalka H, et al. (2013). Transcriptional regulation and specification of neural stem cells. Adv. Exp. Med. Biol. 786: 129-155.

Cuconati A, Mukherjee C, Perez D and White E (2003). DNA damage response and MCL-1 destruction initiate apoptosis in adenovirus-infected cells. Genes Dev. 17: 2922-2932.

D'Arca D, Zhao X, Xu W, Ramirez-Martinez NC, et al. (2010). Huwe1 ubiquitin ligase is essential to synchronize neuronal and glial differentiation in the developing cerebellum. Proc. Natl. Acad. Sci. U. S. A. 107: 5875-5880.

Dantuma NP and Lindsten K (2010). Stressing the ubiquitin-proteasome system. Cardiovasc. Res. 85: 263-271.

Fasanaro P, Capogrossi MC and Martelli F (2010). Regulation of the endothelial cell cycle by the ubiquitin-proteasome system. Cardiovasc. Res. 85: 272-280.

Givogri MI, de Planell M, Galbiati F, Superchi D, et al. (2006). Notch signaling in astrocytes and neuroblasts of the adult subventricular zone in health and after cortical injury. Dev. Neurosci. 28: 81-91.

Giza CC, Kolb B, Harris NG, Asarnow RF, et al. (2009). Hitting a moving target: Basic mechanisms of recovery from acquired developmental brain injury. Dev. Neurorehabil. 12: 255-268.

Hall JR, Kow E, Nevis KR, Lu CK, et al. (2007). Cdc6 stability is regulated by the Huwe1 ubiquitin ligase after DNA damage. Mol. Biol. Cell 18: 3340-3350.

Herold S, Hock A, Herkert B, Berns K, et al. (2008). Miz1 and HectH9 regulate the stability of the checkpoint protein, TopBP1. EMBO J. 27: 2851-2861.

Jiang X, Litkowski PE, Taylor AA, Lin Y, et al. (2010). A role for the ubiquitin-proteasome system in activity-dependent presynaptic silencing. J. Neurosci. 30: 1798-1809.

Kim HC and Huibregtse JM (2009). Polyubiquitination by HECT E3s and the determinants of chain type specificity. Mol. Cell Biol. 29: 3307-3318.

Kinyamu HK, Chen J and Archer TK (2005). Linking the ubiquitin-proteasome pathway to chromatin remodeling/ modification by nuclear receptors. J. Mol. Endocrinol. 34: 281-297.

Makiniemi M, Hillukkala T, Tuusa J, Reini K, et al. (2001). BRCT domain-containing protein TopBP1 functions in DNA replication and damage response. J. Biol. Chem. 276: 30399-30406.

Maspero E, Valentini E, Mari S, Cecatiello V, et al. (2013). Structure of a ubiquitin-loaded HECT ligase reveals the molecular basis for catalytic priming. Nat. Struct. Mol. Biol. 20: 696-701.

Matsuda T, Vande Berg BJ, Bebenek K, Osheroff WP, et al. (2003). The base substitution fidelity of DNA polymerase beta-dependent single nucleotide base excision repair. J. Biol. Chem. 278: 25947-25951. 
Meller R, Thompson SJ, Lusardi TA, Ordonez AN, et al. (2008). Ubiquitin proteasome-mediated synaptic reorganization: a novel mechanism underlying rapid ischemic tolerance. J. Neurosci. 28: 50-59.

Meller R (2009). The role of the ubiquitin proteasome system in ischemia and ischemic tolerance. Neuroscientist 15: 243-260.

Mizutani K, Yoon K, Dang L, Tokunaga A, et al. (2007). Differential Notch signalling distinguishes neural stem cells from intermediate progenitors. Nature 449: 351-355.

Omar Chohan M, Iqbal K, Yonas H and Nemoto E (2013). 163 Enhancement of adult hippocampal neurogenesis by a neurotrophic peptide in traumatic brain injury. Neurosurgery 60 (Suppl 1): 174.

Oya S, Yoshikawa G, Takai K, Tanaka JI, et al. (2009). Attenuation of Notch signaling promotes the differentiation of neural progenitors into neurons in the hippocampal CA1 region after ischemic injury. Neuroscience 158: 683-692.

Palop JJ and Mucke L (2010). Amyloid-beta-induced neuronal dysfunction in Alzheimer's disease: from synapses toward neural networks. Nat. Neurosci. 13: 812-818.

Parsons JL, Tait PS, Finch D, Dianova II, et al. (2009). Ubiquitin ligase ARF-BP1/Mule modulates base excision repair. EMBO J. 28: 3207-3215.

Redmond L, Oh SR, Hicks C, Weinmaster G, et al. (2000). Nuclear Notch1 signaling and the regulation of dendritic development. Nat. Neurosci. 3: 30-40.

Schwarting S, Litwak S, Hao W, Bahr M, et al. (2008). Hematopoietic stem cells reduce postischemic inflammation and ameliorate ischemic brain injury. Stroke 39: 2867-2875.

Valera AG, Diaz-Hernandez M, Hernandez F, Ortega Z, et al. (2005). The ubiquitin-proteasome system in Huntington's disease. Neuroscientist 11: 583-594.

Wang W, Bu B, Xie M, Zhang M, et al. (2009). Neural cell cycle dysregulation and central nervous system diseases. Prog. Neurobiol. 89: 1-17.

Willis MS, Townley-Tilson WH, Kang EY, Homeister JW, et al. (2010). Sent to destroy: the ubiquitin proteasome system regulates cell signaling and protein quality control in cardiovascular development and disease. Circ. Res. 106: 463478.

Yamauchi T, Sakurai M, Abe K, Matsumiya G, et al. (2008). Ubiquitin-mediated stress response in the spinal cord after transient ischemia. Stroke 39: 1883-1889.

Zhao X, D'Arca D, Lim WK, Brahmachary M, et al. (2009). The N-Myc-DLL3 cascade is suppressed by the ubiquitin ligase Huwe1 to inhibit proliferation and promote neurogenesis in the developing brain. Dev. Cell 17: 210-221.

Zhong Q, Gao W, Du F and Wang X (2005). Mule/ARF-BP1, a BH3-only E3 ubiquitin ligase, catalyzes the polyubiquitination of Mcl-1 and regulates apoptosis. Cell 121: 1085-1095. 